\title{
Efectividad de un módulo de resolución de problemas matemáticos en estudiantes de secundaria del Callao
}

\section{Effectiveness of a problem solving model in secondary students in Callao}

\author{
Gloria Fabián $^{1 \mathrm{a}}$ \\ ${ }^{1}$ Universidad Nacional Mayor de San Marcos, Lima, Perú.

\begin{abstract}
${ }^{a}$ Educadora y Magister en Educación con Mención en Aprendizaje y Desarrollo Humano por la Universidad San Ignacio de Loyola. Docente de la Unidad de Post Grado en Educación de la Universidad Nacional Mayor de San Marcos.
\end{abstract}

Recibido: 06-02-13

Aprobado: $25-08-13$

*Correspondencia

Email: propositosyrepresentaciones@usil.edu.pe

\section{Citar Como:}

Fabián, G. (2013). Efectividad de un módulo de resolución de problemas matemáticos en estudiantes de secundaria del Callao. Propósitos y Representaciones, 1(1), 87-105. doi: http://dx.doi.org/10.20511/pyr2013.v1n1.8 


\section{Resumen}

Este trabajo describió y evaluó la aplicación de un módulo para mejorar las capacidades específicas que utiliza el estudiante en la resolución de problemas matemáticos. Tales capacidades son: analizar el problema, identificar y plantear estrategias; aplicar algoritmos y revisar el proceso de resolución. En el módulo se presenta problemas resueltos por el docente (estrategias de modelamiento) y se emplea el trabajo de pares, con el propósito de consolidar y evaluar el trabajo realizado. En la investigación se empleó un diseño cuasiexperimental, con dos grupos pre definidos, con pre-prueba y post-prueba. La muestra fue no probabilística y estuvo conformada por 70 estudiantes. Se utilizó como instrumento una prueba con 10 problemas matemáticos, donde el estudiante debía utilizar las cuatro estrategias propuestas en el módulo. Los resultados muestran diferencias en el rendimiento en matemática a favor del grupo experimental, que nos permiten concluir que el módulo influyó en el desarrollo de las capacidades evaluadas.

Palabras claves: módulo para la resolución de problemas matemáticos, problemas matemáticos, capacidades.

\section{Summary}

This work describes and evaluates the application of a module to increase the specific abilities set in action when students resolve math problems. Such abilities are: analyzing the problem, identifying and laying out strategies, applying algorithms and revising the process that leads to the solution. The module presents problems solved by the teacher (modelling strategies) and uses the work of peers in order to consolidate and evaluate the work. The study uses a quasi experimental design with two pre defined groups, pre and post test. The sample was not probabilistic and was formed by 70 students. A test with 10 math problems was used as the instrument meant for the students to use the four strategies suggested in the module. Results show a difference in favor of the experimental group, which allows us to conclude that the module has an influence in the evaluated abilities.

Key words: math problem solving, analysis of maths problems, problem solving strategies. 
Desde el año 2006 el Ministerio de Educación (MINEDU), a través de la Unidad de Medición de la Calidad Educativa (UMC), inició la evaluación anual a los estudiantes de escuelas públicas y privadas con el propósito de ofrecer a actores educativos importantes información relevante y confiable sobre los aprendizajes en áreas clave (matemática y comprensión lectora) y los factores asociados a los mismos. Ello tenía por finalidad mejorar los procesos de toma de decisiones en las diferentes instancias del sistema educativo peruano (UMC, 2011).

En la Evaluación Censal de Estudiantes (ECE) 2011, para niños de $2^{\circ}$ grado de primaria, solo el 13,2\% lograron un nivel suficiente (Nivel 2) en los aprendizajes en matemática. El 35,8\% se ubicó en el Nivel 1 y el 51\% por debajo del Nivel 1. De cada 10 niños solo uno logró el nivel suficiente en el desarrollo de las capacidades matemáticas relacionadas con la comprensión del número, las operaciones y la resolución de problemas (UMC, 2011).

Lograr que nuestros estudiantes alcancen los niveles de aprendizaje esperados en matemática es un problema que aún no encuentra solución. Ello a pesar de los esfuerzos desplegados por el MINEDU a través de diferentes acciones, como programas de capacitación a docentes o entrega de material educativo a estudiantes.

Entre las causas que estarían explicando esta situación podría señalarse que un porcentaje de las capacidades establecidas en el currículo no son desarrolladas en el aula, o que las capacidades más desarrolladas suelen ser las trabajadas de manera operativa, es decir, mediante tareas de baja demanda cognitiva, situaciones que estarían limitando las oportunidades de aprendizaje de los estudiantes. Estos hechos podrían derivar (o ya vienen derivando) en una enseñanza descontextualizada, alejada de las situaciones de la vida real, que prioriza el uso de algoritmos y un abordaje superficial de los conocimientos. Una enseñanza desvinculada de los problemas (y sus soluciones) cotidianos del estudiante y con resultados poco significativos.

Actualmente la investigación educativa ha dirigido parte de su atención al proceso de enseñanza y aprendizaje de la resolución de problemas, considerado de gran importancia en la intención que los estudiantes lleguen a experimentar sus potencialidades y la utilidad de la matemática para el 
mundo que les rodea (PISA, 2001). La resolución de problemas es reconocida como la actividad cognitiva más importante en el campo de la matemática, tanto por la teoría como por la práctica educativa.

En el proceso de pensamiento, al resolver un problema se aplican conocimientos previos a situaciones nuevas o poco conocidas, asimismo se reorganiza datos y conocimientos previos en un proceso secuencial. En ese sentido, son tan importantes los procedimientos y métodos empleados como el resultado final.

La resolución de problemas es también un tema central en otras disciplinas científicas y se justifica por su aplicación en la vida real. La resolución de problemas es considerada una herramienta para enfrentar diversas situaciones, que cumple, entre otras funciones importantes, las de proporcionar experiencia directa sobre los fenómenos; permitir contrastar la abstracción con la realidad; producir la familiarización de los estudiantes con importantes elementos de carácter tecnológico, desarrollando su competencia técnica; y desarrollar el razonamiento práctico (Barberá y Valdés, 1996, citado por Cabrera, 2003).

La presente investigación busca validar un módulo de aprendizaje de solución de problemas matemáticos para estudiantes secundarios, que se enfoca en el desarrollo de las capacidades de análisis, elaboración, ejecución y revisión en el marco de los procesos de resolución. Con ese fin, la organización del módulo puesto a prueba permite al estudiante guiar su trabajo en el proceso de resolución.

En la investigación sobre la efectividad de los módulos formativos en el desarrollo de la capacidad de resolución de problemas, se distinguen tres líneas de trabajo: la primera, pone énfasis en el medio utilizado (al que muchos autores consideran exclusivamente como un material impreso); la segunda, se enfoca en las formas de presentación elegidas (niveles de lenguaje, instrucciones e información general suministrada, así como los componentes constitutivos); y la tercera trabaja sobre las estrategias metodológicas tenidas en cuenta. El módulo debe ser diseñado de tal forma que llegue a ser un material autodidáctico y autosuficiente, de modo que el estudio de su contenido permita al alumno alcanzar los objetivos de aprendizaje, sin 
necesidad de interactuar con un agente educativo (profesor, tutor, facilitador) cualquiera este sea (Arboleda, 1991; citado por Quiroz, 2001). Definimos al módulo como: "Un conjunto coherente de experiencias de enseñanzaaprendizaje diseñadas para que los estudiantes puedan lograr por sí mismos un conjunto de objetivos interrelacionados" (Quiroz, 2001, p.29).

De acuerdo a Gagné (1992), los módulos deben planificarse cuidadosamente y considerar para ello estos tres puntos: contener objetivos de ejecución claramente especificados y en términos que los estudiantes puedan entender; incluir una evaluación adecuada de la ejecución, para asegurarse que se ha logrado la capacidad especificada en el objetivo; y, contener los materiales necesarios para presentar los acontecimientos didácticos y estimular la memorización de las capacidades o informaciones requeridas.

Según Novak (1996), el diseño de los módulos autoinstructivos debe tomar como base los principios de "actividad" y de "individualización", haciendo que promuevan en el estudiante atención sobre los siguientes aspectos: qué es lo que va a aprender, porqué necesita aprenderlo, cómo lo va a aprender, cómo se dará cuenta de su progreso en el aprendizaje y cuándo estará completo su aprendizaje. Todo ello puede ser traducido en contenidos, justificación, metodología, retroalimentación y logro de objetivos de aprendizaje.

Tomando como referencia el trabajo de García (1994), citado por Quiroz (2001), que reúne investigaciones realizadas sobre el uso de los manuales autoinstructivos, el modelo empírico de módulo considera tres principios básicos: primero, debe enseñar, explicar, animar, preguntar, motivar e informar, ya que sustituye las funciones del profesor y del compañero de clase; segundo, debe contener lecturas, indicar tareas y evaluar todos los procesos; y, tercero, debe enseñar lo esencial de la materia, así como habilidades y actitudes para alcanzar los objetivos de un modo económico y efectivo.

En el diseño del módulo de resolución de problemas matemáticos se consideró los aportes de Ausubel, Novak, y Hanesian (1976), quienes manifiestan que todo aprendizaje significativo depende de dos factores: la naturaleza del material a aprender y la estructura cognoscitiva del estudiante. 
El primero, debe tener un significado lógico para que pueda ser relacionado intencional y sustancialmente con las ideas pertinentes y correspondientes que se hallan dentro del dominio de la capacidad humana. El segundo, se refiere a la disponibilidad y a otras propiedades importantes de los contenidos pertinentes en la estructura cognoscitiva. En esta disponibilidad influyen los conocimientos que posee el estudiante por sus experiencias personales, que actúan facilitando el aprendizaje a lo largo del proceso.

Las actividades propuestas en los módulos deben programarse teniendo en cuenta que el desarrollo de la capacidad de resolución de problemas difiere entre las personas. Estas diferencias están basadas en procesos cognitivos y organizaciones mentales que caracterizan su capacidad para resolver problemas. Hay dos factores importantes que influyen en la resolución de problemas: la naturaleza de la tarea y el conocimiento que las personas tienen en relación al problema (Stemberg, 1982). De acuerdo al segundo factor, Sadovsky (1998), citado por SERCE (2004), plantea que para resolver problemas el alumno debe contar con una serie de aprendizajes esenciales: interpretar la información que se le brinda; seleccionar la información necesaria para responder las preguntas y organizarla; hacer una representación de la situación; movilizar las herramientas matemáticas necesarias; planificar una estrategia de resolución; registrar los procedimientos utilizados; rechazar procedimientos que parecen no conducir a la meta; analizar la razonabilidad de los resultados; validar el procedimiento utilizado y analizar la economía de la estrategia elegida.

Según Sternberg (1986), en la escuela estimulamos diferentes tipos de capacidades cognitivas: las analíticas (comparar, contrastar, analizar, argumentar, criticar); las creativas (elaborar, inventar, imaginar, diseñar, anticipar); y las prácticas (aplicar, manipular, utilizar, demostrar). Este autor manifiesta que la acción de razonar bien consiste en un conjunto de habilidades de razonamiento utilizadas para resolver problemas académicos y cotidianos.

Mayer (1983) también considera a la resolución de problemas como una actividad cognitiva. Utiliza indistintamente a lo largo de su estudio los términos pensamiento, cognición y resolución de problemas. Sostiene que el pensamiento es lo que sucede cuando una persona resuelve un problema, es 
decir, produce un comportamiento que mueve al individuo desde un estado inicial a un estado final o, al menos, trata de lograr ese cambio.

Asimismo, Gagñé (1992) considera que en el aprendizaje se utiliza diferentes tipos de capacidades: habilidades intelectuales, estrategias cognitivas, información verbal, destrezas motoras y actitudes. Las habilidades intelectuales son las más complejas y se componen de procesos más simples: discriminaciones, conceptos concretos y definidos, reglas simples y de orden superior, que suelen aprenderse al resolver problemas. Las reglas de orden superior son las más complejas y tienen como objeto resolver un problema o una clase de problemas prácticos. El estudiante al dar una solución práctica a un problema adquiere también una nueva capacidad. La regla recién aprendida será almacenada en la memoria y se usará nuevamente para resolver otros problemas, y de esta manera se irá combinando reglas importantes para formar una nueva regla de orden superior.

Según Anderson (1983), citado por Pozo y Postigo (1994), las estrategias de solución de problemas serían procedimientos que se aplican, de modo intencional y deliberado, a una tarea que no podría reducirse a rutinas automatizadas. Así, la formulación y comprobación de hipótesis es, sin duda, un conjunto de procedimientos que sólo puede aplicarse de modo consciente. Dentro de los procedimientos que los alumnos deben adquirir para resolver problemas, algunos consisten en técnicas o rutinas que deben automatizar (por ejemplo, la conversión de unidades de medida de un sistema a otro o la decodificación de una gráfica o una tabla), mientras que otros requieren planificación y control en su ejecución (por ejemplo, el diseño de un experimento o la búsqueda de fuentes de información, para contrastar la explicación de un fenómeno social o histórico). Existiría, por tanto, dos rutas para el aprendizaje, no necesariamente incompatibles o contradictorias.

Como han sugerido varios autores, la adquisición de la pericia en un área puede basarse bien en el dominio rutinario de técnicas o destrezas, o en otro más consciente o significativo de esas destrezas, que permita su adaptación y generalización a nuevas situaciones de aprendizaje. Esas dos formas de ser experto constituyen, a su vez, dos formas distintas de adquirir el conocimiento procedimental. Sin embargo, no son igualmente eficaces a la hora de aprender a resolver problemas. En el primer caso, nos hallaremos 
ante un dominio rutinario de técnicas y destrezas, útil para resolver ejercicios, pero no problemas. En el segundo, ante un uso más controlado y planificado de esas mismas técnicas con fines estratégicos. Este último se halla vinculado a las estrategias de solución de problemas.

Polya (1966), citado por Chavarría y Alfaro (2009), brinda un aporte significativo a la enseñanza de la matemática, en particular, a la resolución de problemas. Muestra cómo la construcción matemática puede ser aprovechada para su enseñanza, es decir, cómo las estrategias seguidas por un profesional en matemática, que Polya denomina "razonamientos plausibles", pueden permitirle a un estudiante aprender matemáticas.

Schoenfeld (1985), citado por Chavarría y Alfaro (2009), considera insuficientes las estrategias planteadas por Polya para la resolución de problemas. Sostiene que este proceso es más complejo e involucra más elementos que inclusive son de carácter emocional-afectivo, psicológico, sociocultural, entre otros. Propone la existencia de cuatro aspectos que intervienen en el proceso de resolución de problemas: los recursos (entendidos como conocimientos previos, o bien el dominio del conocimiento), las heurísticas (estrategias cognitivas), el control (estrategias metacognitivas) y el sistema de creencias. Como parte de las estrategias metacognitivas se considera las de planificación, regulación y control del proceso de resolución.

En la línea de desarrollo de las ideas de Polya, Schoenfeld (1985), citado por Chavarría y Alfaro (2009), ofrece una lista de técnicas heurísticas de uso frecuente que se agrupa en tres fases:

- Análisis: trazar un plan, examinar casos particulares y probar la simplificación del problema.

- Exploración: examinar problemas esencialmente equivalentes, problemas ligeramente modificados y problemas ampliamente modificados.

- Comprobación de la solución obtenida: verificar la solución con criterios específicos (a través de las siguientes preguntas: ¿utiliza todos los datos pertinentes?, ¿está acorde con predicciones o estimaciones razonables?) y criterios generales (a través de las siguientes preguntas: ¿es posibles obtener la misma solución por otro método?, ¿puede concretarse en el 
caso particular?, ¿es posible reducirla a resultados conocidos?, ¿es posible utilizarla para generar algo ya conocido?).

Asimismo, diversas investigaciones en educación buscan afirmar los aportes teóricos propuestos por los autores antes mencionados. Gascón (1989) se propuso contribuir al desarrollo de una teoría de resolución de problemas de matemáticas, dentro de la tradición heurística reinaugurada por Polya. Arlandis (1992) buscó comprobar la efectividad diferencial en la aplicación de las técnicas cognitivo comportamentales en el tratamiento de la resolución de problemas de matemática. Fregoso (1987) presentó un curso desarrollado y sistematizado en unidades temáticas, cada una de las cuales incorporaba una serie de elementos de aprendizaje bajo los principios de "actividad" y de "individualización", concluyendo que el empleo de los materiales autoinstructivos permite a los profesores realizar una labor de verdaderos coordinadores, evitando dedicar todo su tiempo al dictado de clases.

Otro grupo de investigadores se encargaron de diseñar e implementar modelos que permiten el desarrollo de la capacidad de resolución de problemas. Pifarré y Sanuy (2001) se propusieron diseñar e implementar un proceso de enseñanza que amplíe y mejore el repertorio de estrategias de los alumnos para resolver problemas de proporcionalidad directa. En los resultados se observó un incremento estadísticamente significativo respecto al nivel de aprendizaje inicial, que permitía afirmar que la propuesta didáctica tenía incidencia positiva en el proceso de aprendizaje. Vicente, Orrantia y Lieven (2002) realizaron un estudio con el propósito de incorporar en el proceso de resolución de problemas dos estrategias: la reescritura del problema y la realización de gráficos (esquemas). Los resultados mostraron que la reescritura y los dibujos matemáticos incrementan el acierto, especialmente para los alumnos más competentes. Fernández (2006) aplicó y evaluó un programa de invención-reconstrucción de situaciones problemáticas para mejorar el rendimiento en la resolución de problemas matemáticos, concluyendo que la exposición de técnicas y destrezas pone énfasis en la creatividad con la que se desenvuelven los estudiantes en la construcción y resolución de problemas matemáticos. Por último, Vílchez (2007) elaboró un módulo didáctico para la enseñanza de las funciones trigonométricas, a partir de la circunferencia unitaria en el plano cartesiano, formulando la hipótesis de que su implementación en el proceso de enseñanza permite un aprendizaje más efectivo. Concluyó que la enseñanza personalizada con el 
módulo didáctico motiva y desarrolla actitudes positivas para el aprendizaje individual y grupal de los alumnos.

De acuerdo con el análisis bibliográfico efectuado, se planteó la siguiente hipótesis general: si se aplica el módulo "Hacia la Meta" en un grupo de estudiantes, su capacidad de resolución de problemas se elevará. Esta conjetura se desprende en dos hipótesis específicas:

1. Si se aplica el módulo "Hacia la Meta" en un grupo de estudiantes, entonces las capacidades de analizar problemas e identificar y plantear estrategias se elevarán.

2. Si se aplica el módulo "Hacia la Meta" en un grupo de estudiantes, entonces las capacidades específicas de aplicar algoritmos y revisar el proceso de resolución se elevarán.

\section{Método}

El diseño del estudio fue de tipo cuasi-experimental, con pre-prueba y postprueba aplicadas en grupos intactos, pues este tipo de diseño tiene como propósito conocer la efectividad de las intervenciones (Hernández, Fernández y Baptista, 2010).

El universo estuvo conformado por 300 estudiantes, varones y mujeres, del primer año de secundaria de la Institución Educativa "Sor Ana de los Ángeles", con un rango de edades de 12 a 14 años. La muestra empleada fue no probabilística y asignó intencionalmente el 10 "F" al grupo experimental y el 1 o " $\mathrm{H}$ " al grupo de control.

Entre los criterios de inclusión/exclusión se consideró los siguientes: los estudiantes debían tener conocimientos previos de las operaciones aritméticas (adición, sustracción, multiplicación, división, potenciación y radicación). Esta variable fue medida por una prueba al inicio del experimento (pre-prueba), cuyos promedios fueron homogéneos. También se consideró la puntualidad y asistencia a clases en ambos grupos, excluyendo a los estudiantes con el $30 \%$ de inasistencia o más.

La intervención empleó el módulo de resolución de problemas matemáticos diseñado a partir de la propuesta de Novak (1996). El módulo 
se organizó en tres capítulos, compuesto cada uno de introducción, objetivos y metodología. El trabajo realizado puede describirse de la siguiente manera:

- Capítulo 1: presenta la "hoja para pensar el problema" con los cuatro pasos de Polya (1945) y las preguntas de reflexión que el estudiante debe utilizar en todo proceso de resolución de problemas.

- Capítulo 2: compete a problemas de "número, relaciones y funciones". En un primer momento se hizo uso de la demostración del proceso de resolución de problemas que los estudiantes deben utilizar, hecha sobre un problema concreto y tratando que reestructurar el proceso ya adquirido en el nivel primario (Sternberg, 1986). Luego, el docente y los estudiantes resuelven conjuntamente otro problema, siguiendo las pautas anteriores, aclarando situaciones y despejando dudas en los estudiantes. Por último, los estudiantes son organizados para trabajar en grupos de a dos (en forma colaborativa) y el docente asigna problemas iguales (dentro de aquellos problemas que forman parte del módulo) a cada dos grupos. Finalmente, dichos problemas son expuestos, poniendo énfasis en la variabilidad del procedimiento al resolverlos.

- Capítulo 3: compete a problemas de "geometría y medición" y empleó el mismo método de trabajo del capítulo 2.

El instrumento empleado en el estudio fue una prueba de resolución de problemas para evaluar el proceso cognitivo que utiliza el estudiante al momento de resolver problemas. Se observa cuatro dimensiones: análisis del problema, identificación y planteamiento de la estrategia, aplicación de algoritmos y revisión del proceso de resolución. La prueba consta de 10 problemas con respuesta abierta, cinco pertenecen al componente "número, relaciones y funciones" y cinco al componente "geometría y medición". Ambos componentes propuestos en el Diseño Curricular Básico por el MINEDU.

Para la evaluación de los problemas que contenía la prueba se elaboró una ficha estructurada sobre cuatro dimensiones: análisis del problema; identificación y planteamiento de la estrategia; aplicación de los algoritmos y revisión del proceso de resolución. Cada dimensión, a su vez, estaba compuesta de tres indicadores, como se muestra: 
- Dimensión 1: no reconoce los datos ni la incógnita; reconoce y anota algunos de los datos; reconoce y anota todos los datos y la incógnita.

- Dimensión 2: establece una estrategia equivocada; establece una estrategia pero incompleta, y establece una estrategia evidenciando un orden lógico y completo.

- Dimensión 3: no establece algoritmo alguno/ejecuta algoritmos equivocados; ejecuta los algoritmos establecidos en la estrategia de manera desordenada e incompleta, y ejecuta los algoritmos establecidos en la estrategia de manera ordenada y completa.

- Dimensión 4: no escribe la respuesta del problema; escribe la respuesta incompleta del problema, y escribe la respuesta del problema de manera clara y completa.

La prueba fue sometida al criterio de cinco jueces expertos en matemática, a quienes se solicitó verificar si los ítems que la integran constituyen una muestra representativa de los indicadores de la propiedad que se mide (Sánchez, 2006). Para ello, se utilizó el coeficiente $V$ de Aiken con fines de establecer niveles de validez de contenido.

Posteriormente se aplicó un estudio piloto con 30 estudiantes de la misma institución y de similares características a quienes conformaron la muestra definitiva. El propósito fue verificar la comprensión de los reactivos de la prueba con el uso del coeficiente alfa de Cronbach. El resultado arrojó un índice de .742, dentro de los parámetros de confiabilidad.

\section{Resultados}

Para contrastar las hipótesis específicas se obtuvo las puntuaciones del pre-test de ambos grupos, como se explica a continuación y se muestra en la tabla 1 . 
Tabla 1.

Diferencias grupales de las dimensiones de la capacidad de la resolución de problemas de la pre-prueba

\begin{tabular}{lcccccc}
\hline & \multicolumn{4}{c}{ Grupo control } & \multicolumn{3}{c}{ Grupo experimental } & \\
\cline { 2 - 5 } Dimensiones & $\mathrm{M}$ & $\mathrm{DE}$ & $\mathrm{M}$ & $\mathrm{DE}$ & $\mathrm{t}$ \\
\hline Analizar el problema & 4.4571 & 3.05193 & 5.31 & 2.398 & -1.306 \\
Identificar y plantear estrategia & 3.3143 & 1.93682 & 4.71 & 3.536 & -2.054 \\
Aplicar algoritmos & 3.3143 & 1.93682 & 4.63 & 3.565 & -1.912 \\
Revisar el proceso de resolución & 2.5143 & 1.91544 & 4.00 & 3.387 & -2.259 \\
\hline $\mathrm{N}=35$ & & & & & &
\end{tabular}

Los valores promedio y desviación para la dimensión "analizar el problema" de la pre-prueba en el grupo control fueron 4.46 (3.05), que difieren de los del grupo experimental que fueron 5.31 (2.40). La prueba $t$ de student arrojó un valor de $-1.306(g l=68)$, significativo al .196 (en un nivel de significación $p<.05$ ), que nos llevó a sostener que no existían diferencias significativas entre los grupos en el momento de la pre-prueba.

En la dimensión "identificar y plantear estrategia" las puntuaciones fueron 3.31 (1.93) para el grupo de control y 4.71 (3.53) para el grupo experimental. La $t$ de student arrojó un valor de $-2.054(g l=68)$, significativo al .044 (en un nivel de significación $p<.05$ ), que establece diferencias significativas entre los grupos en relación a esta dimensión.

En la dimensión "aplicar algoritmos" las puntuaciones fueron de, 3.31 (1.93) en el grupo control y 4.63 (3.56) para el grupo experimental. La $t$ de student arrojó un valor de $-1.912(g l=68)$, significativo al .60 (en un nivel de significación $p<.05$ ), que nos llevó a sostener que no existían diferencias significativas entre los grupos en el momento de la pre-prueba.

Las hipótesis específicas planteadas se contrastan con los resultados de la tabla 2, que muestra el análisis comparativo de los valores promedio de la postprueba del grupo experimental y control. Se aprecia que existen diferencias 
significativas en las dimensiones de la capacidad de resolución de problemas. Con respecto a la dimensión "analizar el problema" existen diferencias significativas $(\mathrm{t}=-5.595 \mathrm{p}>.05)$. Lo mismo ocurre con las dimensiones "Identificar y plantear estrategias" ( $\mathrm{t}=-2.423 \mathrm{p}>.05)$; "aplicar algoritmos" $(\mathrm{t}$ $=-2.500 \mathrm{p}>.05) ; \mathrm{y}$, por último, "revisar el proceso de resolución" $(\mathrm{t}=-3.265$ $\mathrm{p}>.05)$. Estos datos nos llevan a aceptar las hipótesis específicas planteadas.

Tabla 2.

Diferencias de los promedios de las dimensiones de la capacidad de la resolución de problemas de la post-prueba del grupo control con el grupo experimental.

\begin{tabular}{|c|c|c|c|c|c|}
\hline \multirow[t]{2}{*}{ Dimensiones } & \multicolumn{2}{|c|}{ Grupo control } & \multicolumn{2}{|c|}{ Grupo experimental } & \multirow[b]{2}{*}{$\mathrm{t}$} \\
\hline & $M$ & DE & $M$ & DE & \\
\hline Analizar el problema & 5.3714 & 2.66884 & 10.0857 & 4.21043 & -5.595 \\
\hline $\begin{array}{l}\text { Identificar y plantear } \\
\text { estrategia }\end{array}$ & 3.8857 & 2.90812 & 5.8857 & 3.92407 & -2.423 \\
\hline Aplicar algoritmos & 3.8000 & 2.76320 & 5.7714 & 3.75802 & -2.500 \\
\hline $\begin{array}{l}\text { Revisar el proceso de } \\
\text { resolución }\end{array}$ & 2.6286 & 2.57917 & 5.1143 & 3.69237 & -3.265 \\
\hline
\end{tabular}

Respecto a la hipótesis general, el resultado de la tabla 3 permite apreciar que hay diferencias estadísticas significativas $(t=-3.659 \mathrm{p}>.05)$ en el puntaje promedio entre la post-prueba del grupo control y del grupo experimental. En conclusión, la hipótesis general prevista en el estudio es sostenible.

Tabla 3.

Diferencia de los promedios de la capacidad de la resolución de problemas de la post-prueba del grupo control y del grupo experimental

\begin{tabular}{lccccc}
\hline Prueba & \multicolumn{4}{c}{ Grupo control } & \multicolumn{3}{c}{ Grupo experimental } & \\
\cline { 2 - 5 } & $\mathrm{M}$ & $\mathrm{DE}$ & $\mathrm{M}$ & $\mathrm{DE}$ & $\mathrm{t}$ \\
\hline Post.prueba & 15.63 & 10.152 & 26.83 & 14.521 & -3.659 \\
\hline
\end{tabular}




\section{Discusión}

Se aprecia que la hipótesis general y las hipótesis específicas son aceptadas. Los resultados brindan cierta seguridad sobre la contribución del módulo en mejorar los mecanismos mentales que dirigen los comportamientos de los estudiantes al momento de resolver problemas matemáticos. Las hipótesis confirman, de esta manera, lo propuesto por la teoría triárquica de la inteligencia humana de Sternberg (1986), a la vez de ratificar un aumento significativo en la capacidad de resolución de problemas después de la aplicación del módulo, como se ha propuesto en las tres dimensiones de dicha teoría (componencial, experimental, contexto). De ello se desprendería que la resolución de problemas es una actividad cognitiva como lo propuso Mayer (1983).

Por otro lado, las estrategias que se propusieron en el módulo, de modo intencional y deliberado, permitieron elevar las medias entre la pre-prueba y post-prueba. Estos resultados ratifican lo hallado por Anderson (1983), citado por Pozo (1994), y Polya (1945), citado por Vilanova (2009). Es presumible que un elemento importante en la explicación de los resultados sea la incorporación de estrategias de carácter emocional-afectivo, psicológico y social-cultural.

Además, por la consistencia de los hallazgos de este estudio con los hallazgos de Arlandis (1992), Gascón (1989), Ibáñez (2004), Mesia (1993) y Viíchez (2007), quienes también encontraron niveles de efectividad de los módulos de metodologías y técnicas en la resolución de problemas, se podría contribuir a sustentar que la enseñanza con módulos didácticos motiva y desarrolla actitudes positivas para el aprendizaje individual y grupal de los estudiantes al momento de resolver problemas matemáticos.

Sin embargo, a pesar de que la autora consideró, en el módulo y la pruebas, problemas acordes a la realidad del estudiante, estructurados según su capacidad cognitiva y la disponibilidad de conocimientos previos para facilitar el aprendizaje, tomando como referencia los aportes de Ausubel et al. (1976), es posible presumir limitaciones de tiempo en la aplicación. La programación del módulo con 40 horas pedagógicas resultó insuficiente para que el estudiante pudiera abarcar todo lo relativo a la metodología de resolución de problemas. Un efecto de ello puede presentarse en los puntajes alcanzados por ambos grupos (experimental y control) en la post-prueba, 
porque si bien el grupo experimental alcanza una media significativamente mayor al grupo de control, ambos puntajes se ubican por debajo de lo aceptado como óptimo, según el baremo elaborado por la autora. En la misma dirección, Pifarré y Sanuy (2001) han puesto énfasis en la importancia del factor tiempo en la implementación de un proceso de enseñanza que amplíe y mejore el repertorio de estrategias para resolver problemas de proporcionalidad directa. Como parte de sus resultados, solo lograron que el $50 \%$ de los estudiantes involucrados en el estudio resuelvan correctamente la prueba de evaluación final, habiendo utilizado 30 horas en la intervención.

Además, no debe perderse de vista la importancia de otros aspectos que intervienen en el proceso de enseñanza-aprendizaje en la resolución de problemas matemáticos, como las características de las instituciones, el conocimiento o desempeño de los docentes y el esfuerzo personal desplegado por cada alumno. Uno de esos factores, reportado por Falcón (1995), tiene que ver con la secuencia en que se debe dar el aprendizaje de problemas, en un orden de dificultad que tenga en cuenta la complejidad cognitiva requerida para resolverlos.

\section{Referencias}

Acuerdos de la XIV Reunión de Coordinadores Nacionales para el SERCE, (2004), Buenos Aires.pp.1-11 [en línea] Disponible en: http://hydra. icfes.gov.co/serce/ [Consulta: 11 de julio 2010]

Arlandis, P. (1992). Estudiantes con dificultades en la resolución de problemas de matemáticas, efectos de la instrucción en estrategias y del reentrenamiento atribucional". [en línea]. Disponible en: http://www.cibernetia.com/tesis_es/PSICOLOGIA/PSICOLOGIA_ DEL_NI\%D1O_Y_DEL_ADOLESCENTE/PROBLEMAS_DE_ APRENDIZAJE/1 [Consulta: 15 de enero 2010].

Ausubel, D.P., Novak, J. \& Hanesian, H. (1976). Psicología educativa un punto de vista cognoscitivo. México: Trillas.

Cabrera, G. (2003). La resolución de trabajos prácticos como problemas.

Tesis publicada. Universidad de la Laguna. [en línea] Diisponible en: http://dialnet.unirioja.es/servlet/tesis?codigo $=1036$ [Consulta: 2 de setiembre 2010] 
Chavarría, J., \& Alfaro, C. (2009). Resolución de problemas según Polya y Schoenfeld. Ciemac, IV, 1-4. [en línea] Disponible en: http://www. cidse.itcr.ac.cr/ciemac/4tociemac/ponencias/resoluciondeproblemas.pdf [Consulta: 14 de abril 2010]

Falcón, A. (1995). Efectos de la aplicación de un programa de resolución de problemas matemáticos en el tercer grado de educación primaria de menores. Tesis publicada. Perú. [en línea] Disponible en: http:// www.cybertesis.edu.pe/sdx/sisbib/ [Consulta: 10 de enero 2009]

Fernández, J. (2006). Investigación sobre los efectos de la invenciónreconstrucción de situaciones problemáticas, en el rendimiento de los alumnos para la resolución de problemas matemáticos. Tesis publicada. Madrid. [en línea] Disponible en: dialnet.unirioja.es/ servlet/fichero_articulo?codigo=2043996 [Consulta: 2 de marzo 2010]

Fregoso, M. (1987). Guía para la elaboración de paquetes didácticos. México: Universidad Autónoma de México. [en línea] Disponible en: http://sisbib.unmsm.edu.pe/bibvirtualdata/tesis/human/quiroz_pr/cap2. pdf [Consulta: 14 de junio 2009].

Gagné, R. (1992)- La planificación de la enseñanza. Sus principios. México: Editorial Trillas.

Gascón, J. (1989). El aprendizaje de métodos de resolución de problemas de matemática. Tesis publicada. Universidad Autónoma de Barcelona. España. [en línea] Disponible en: http://dialnet.unirioja.es/servlet/ tesis?codigo $=11073$ [Consulta en: 5 de julio 2010]

Hernández, R; Fernández, C y Baptista, P. (2010) Metodología de la Investigación, México DF: McGraw-Hill.

Ibáñez, M. (2004). Aplicación de una metodología de resolución de problemas como una investigación para el desarrollo de un enfoque ciencia-tecnología-sociedad en el currículo de biología de educación secundaria. Tesis. [en línea] Disponible en: http://dialnet.unirioja.es/ servlet/busquedadoc $? \mathrm{db}=1 \& \mathrm{t}=$ influencia $+\mathrm{del}+$ conocimiento + matem $\% \mathrm{C} 3 \% \mathrm{~A} 1 \mathrm{tico}+\mathrm{y}+$ situacional\&td=todo [Consulta: 15 de enero 2010]

Mayer, R. (1983). Pensamiento, resolución de problemas y cognición. Barcelona: Paidós. 
Mesía, R. (1993). La utilización de módulos autoinstructivos en la enseñanza de la formulación y nomenclura química. Tesis, Uiversidad Nacional Mayor de San Marcos. Perú. [en línea] Disponible en: http://www. cybertesis.edu.pe/sdx/sisbib/ [Consulta: 10 de enero 2009]

Novak, J. (1996) Teoría y práctica de la educación. Madrid: Alianza Editorial.

Pifarré, M. \& Sanuy, J. (2001). La enseñanza de estrategias de resolución de problemas matemáticos en la Eso: un ejemplo concreto. Tesis. Universidad de Lleida. España. [en línea] Disponible en: http://www. raco.cat/index.php/ensenanza/article/viewFile/21745/21579 [Consulta: 15 de enero 2010].

PISA. (2001). Conocimientos y Ddestrezas para la vida: Primeros resultados del Proyecto PISA 2000: Resumen de resultados. Madrid, Ministerio de Educación, Cultura y Deporte. [en línea] Disponible en: www.simce.cl/ index.php?id=100 [Consulta: 12 de setiembre 2009]

Pozo, J. \& Postigo, Y. (1994). La solución de problemas. Madrid: Santillana:

Quiroz, R. (2001). El empleo de módulos autoinstructivos en la enseñanza-aprendizaje de la asignatura de legislación y deontología bibliotecológica. Perú: Tesis, Uiversidad Nacional Mayor de San Marcos. [en línea] Disponible en: http://www.cybertesis.edu.pe/sdx/ sisbib/ [Consulta: 1 de enero 2010]

Sánchez, H. (2006). Metodología y diseños en la investigación científica. Lima: Editorial Visión.

Sternberg, R. (1982). Inteligencia Humana: Cognición. Madrid: Paidós.

Unidad de Medición de la Calidad de la Educación. (2011) Evaluación Censal de Estudiantes - ECE 2010. Lima: MINEDU - UMC.

Vilchez, J. (2007). Modelo de enseñanza modular personalizada de las funciones trigonométricas en el quinto año de secundaria. Tesis, Universidad Nacional Mayor de San Marcos. [en línea] Disponible en: http://www.cybertesis.edu.pe/sdx/sisbib/ [Consulta: 10 de enero 2009]

Vicente, S., Orrantia, J. \& Lieven, V. (2002). Influencia del conocimiento matemático y situacional en la resolución de problemas aritméticos verbales: ayudas textuales y gráficas. Tesis. [en línea] Disponible en: 
http://dialnet.unirioja.es/servlet/busquedadoc? $\mathrm{db}=1 \& \mathrm{t}=$ influencia + del + conocimiento + matem $\% \mathrm{C} 3 \% \mathrm{~A} 1$ tico $+\mathrm{y}+$ situacional\&td=todo [Consulta: 15 de enero 2010]

Vilanova, S. (2009). Argentina: El papel de la resolución de problemas en el aprendizaje. Iberoamérica de Educación, 1-11 [en línea] Disponible en: http://www.rieoei.org/deloslectores/203Vilanova.PDF [Consulta: 14 de abril 2010] 